\title{
Amphiphilic Ionic Liquid Induced Fusion of Phospholipid Liposomes
}

\author{
Sandeep Kumar ${ }^{\#}$, Navleen Kaur ${ }^{\#}$, Venus Singh Mithu ${ }^{\# *}$ \\ \#Department of Chemistry, Guru Nanak Dev University, Amritsar-143005, India
}

\section{AUTHOR INFORMATION}

Corresponding Author:

venus.chem@gndu.ac.in

KEYWORDS. Ionic liquid; Membrane fusion; Fusion-holes; Lipid Splay; solid-state NMR. 
ABSTRACT. We have investigated the impact of increasing concentrations of imidazoliumbased ionic liquids $\left(\left[\mathrm{C}_{\mathrm{n}} \mathrm{MIM}\right]^{+}[\mathrm{Br}]^{-}\right)$on structural integrity of large unilamellar vesicles (LUVs) made of pure phosphatidylcholine (PC) and phosphatidylglycerol (PG) lipids. Calcein based dye leakage assays were used to monitor permeability of LUVs in presence of ionic liquids. As ionic liquid concentration approaches the critical micelle value, vesicle fusion occurs resulting in unexpected quenching which is accompanied by a rapid dye leakage due to formation of transiently lived fusion-holes. Vesicle fusion is confirmed using dynamic light scattering based size measurements and Fluorescence based lipid mixing assays. ${ }^{1} \mathrm{H}-{ }^{1} \mathrm{H}$ NOESY measurements using solid-state NMR spectroscopy were performed to obtain insights into fusion mechanism. While POPC LUVs are more prone to membrane fusion, the overall extent of fusion is higher in POPG. Ionic liquid induced splaying of phospholipid chains is crucial for overcoming the hydration barrier between the merging bilayers. 


\section{INTRODUCTION}

Merging of inner and outer leaflets of two bilayers, also known as membrane fusion, is a key biological phenomenon with potential applications in biotechnology. ${ }^{1}$ It is known to play a crucial role in several biological events such as fertilization, viral infections, cellular trafficking, endocytosis, exocytosis, and muscle development. ${ }^{2-3}$ In vivo, proteins and peptides act as fusogenic agents and assist the process of membrane fusion. ${ }^{4-6}$ In vitro, metals ions, lipids, small organic ligands, polymers, drugs, and surfactants have been shown to act as fusogenic agents. ${ }^{7}$ Mechanistically, protein-free fusion begins with the merging of outer leaflets (OL) of lipid bilayer leading to formation of the stalk. The stalk further evolves into a hemifusion-diaphragm where lipids from the inner leaflets (IL) of the merging bilayers start to mix, and eventually form a fusion pore allowing contents of two liposomes to mix. ${ }^{1,7}$ Fusogens can be classified into two categories; lipid-soluble and water-soluble. Lipid-soluble fusogens act by perturbing the structure of hydrocarbon chain thereby increasing the membrane contact area due to enhanced surface exposure of lipid chains. ${ }^{8-9}$ Water-soluble fusogens, like metal ions, alter the membrane surface potential or hydration of the polar head-groups allowing the fusing membranes to achieve desired proximity. ${ }^{10-11}$ In this context, amphiphilic ionic liquid molecules with their long lyophobic chains and charged head-groups possess properties of both type of fusogens and hence, are interesting candidates to be explored as fusogenic agents.

Ionic liquids are the molten salts with exciting physiochemical properties. ${ }^{12}$ Due to a size mismatch in composing ions, their melting points are considerable lower $\left(\sim 100^{\circ} \mathrm{C}\right)$ than regular ionic salts. ${ }^{12}$ Unique characteristics of ionic liquids include high ionic conductivity, nonvolatility and a wide range of viscosity. ${ }^{12}$ Moreover, their properties can be easily tuned by changing the combination of composing ions. ${ }^{12}$ These properties make ionic liquids acceptable 
as alternative to conventional solvents in chemical processes, and as bioactive agents in pharmaceutical processes. ${ }^{13}$ Widespread application of ionic liquids has prompted the scientific community to test and understand the cytotoxic behaviour of commonly used ionic liquids containing amphiphilic cations. ${ }^{14}$ Cytotoxicity of ionic liquids is rooted in the amphiphilic nature of cations, and is known to correlate directly with length of alkyl chains. ${ }^{15}$ Various computational studies, ${ }^{16-18}$ and investigations based on neutron reflectometry ${ }^{19-20}$ and solid-state $\mathrm{NMR}^{15,21}$ have revealed the structural details of their interaction with lipid bilayers. In this mechanism, the alkyl chain enters the hydrophobic phase (OL of bilayer) while the charged head group resides at the lipid-water interface. ${ }^{15,19-21}$ Insertion of cations in this manner puts the OL under strain ultimately leading to membrane permeabilization and disruption. ${ }^{22} \mathrm{~A}$ similar mechanism could also lead to fusion of vesicles. Eri et al have shown fusion of DMPC+SM (3:1) liposomes under the influence of ionic liquids containing short chain amphiphilic 1-ethyl-3methylimidazolium cations. ${ }^{23}$ In another study, Galletti et al have compared the impact of difference cationic head groups on fusion of egg-PC liposomes. ${ }^{24}$ Apart from these two reports, ionic liquid induced membrane fusion remains unexplored.

In this work, we have studied the impact of $\left[\mathrm{C}_{12} \mathrm{MIM}\right]^{+}[\mathrm{Br}]^{-}$ionic liquids on large unilamellar vesicles (LUVs) made of zwitterionic 1-palmitoyl-2-oleoyl-sn-glycero-3-phosphocholine (POPC) and anionic 1-palmitoyl-2-oleoyl-sn-glycero-3-phospho-(1'-rac-glycerol) (POPG). Fluorescence based dye leakage assays were performed to monitor vesicle permeabilization as a function of ionic liquid concentration. Abrupt changes in dye leakage profiles near critical micelle concentration (CMC) of $\left[\mathrm{C}_{12} \mathrm{MIM}\right]^{+}[\mathrm{Br}]^{-}$indicated that fusion of LUVs is taking place. To confirm further, we monitored size distribution of LUVs using dynamic light scattering (DLS). Lipid mixing assays were performed to determine the mechanism of membrane fusion 
using LUVs containing 1,2-dipalmitoyl-sn-glycero-3-phosphoethanolamine-N-(7-nitro-2-1,3benzoxadiazol-4-yl) (NBD-PE) and 1,2-dioleoyl-sn-glycero-3-phosphoethanolamine-N(lissamine rhodamine B sulfonyl) (Rho-PE) as FRET probes. To gain molecular insights, ionic liquid induced lipid splay was estimated using solid-state NMR spectroscopy. A membrane fusion model has been developed based on these observed. Outcomes of this model were tested using similar ionic liquids with shorter alkyl chains $\left(\left[\mathrm{C}_{8} \mathrm{MIM}\right]^{+}[\mathrm{Br}]^{-}\right)$.

\section{EXPERIMENTAL METHODS}

Materials. Chloroform stock solutions of 1-palmitoyl-2-oleoyl-sn-glycero-3-phosphocholine (POPC), 1-palmitoyl-2-oleoyl-sn-glycero-3-phospho-(1'-rac-glycerol) (sodium salt) (POPG), L$\alpha$-Phosphatidylethanolamine-N-(lissamine rhodamine B sulfonyl) (Ammonium Salt) (Rh-PE), 1,2-dioleoyl-sn-glycero-3-phosphoethanolamine-N-(7-nitro-2-1,3-benzoxadiazol-4-yl)

(ammonium salt) (NBD-PE) were purchased from Avanti Polar Lipids, Inc. (Alabaster, AL) and used without further purification. Sephadex G-50 was purchased from Sigma Aldrich, India. Calcein, Triton X-100, and anhydrous sodium phosphate monobasic (AR grade) were purchased from Sisco Research Laboratories Pvt. Ltd. (Maharashtra, India). 4-amino-3-hydroxy-1naphthalene sulfonic acid (Purity 99.0\%), 1-Bromooctane, 1-bromododecane (Purity 98.0\%), and sodium dithionite (DTN) were purchased from Alfa Aesar, India. Ammonium heptamolybdate tetrahydrate, sodium sulphite anhydrous were purchased from Merck, India. Sodium metabisulfite was purchased from Fisher Scientific, India. 1-Methyl imidazole was purchased from Spectrochem, India. Tris hydrochloride 99\% (Molecular biology grade), Sodium Chloride (AR grade), Sulphuric acid 98\% (AR grade) were purchased from Loba Chemie, Mumbai, India. Diethylether (AR grade), and Sodium hydroxide pellets (AR grade) were purchased from SD 
fine-chem limited, (Mumbai, India). Perchloric acid 70\% (AR grade) was purchased from Qualikems Fine Chem Pvt. Ltd., India. Ionic liquids $\left[\mathrm{C}_{n} \mathrm{MIM}\right]^{+} \mathrm{Br}^{-}(\mathrm{n}=8,12)$ were synthesized as reported previously. ${ }^{15}$

Methods. Dye Leakage Assay. Dye leakage assays were performed on LUVs containing selfquenched calcein dye $(70 \mathrm{mM})$ which were prepared as described previously. ${ }^{15}$ Stock solutions of $\left[\mathrm{C}_{8} \mathrm{MIM}\right]^{+}[\mathrm{Br}]^{-}(1 \mathrm{M})$, and $\left[\mathrm{C}_{12} \mathrm{MIM}\right]^{+}[\mathrm{Br}]^{-}(100 \mathrm{mM})$ were prepared in $7.7 \mathrm{mM}$ Tris $\mathrm{HCl}$ buffer containing $100 \mathrm{mM}$ of $\mathrm{NaCl}$ ( $\mathrm{pH}$ 7.4). Appropriate amount of dye filled LUVs were added to the buffer containing $\left[\mathrm{C}_{\mathrm{n}} \mathrm{MIM}\right]^{+}[\mathrm{Br}]^{-}(\mathrm{n}=8,12)$ achieving a final lipid concentration of $0.275 \pm$ $0.015 \mathrm{mM}$ in each case. The solutions were gently mixed and transferred to quartz cuvette to perform fluorescence measurements (dead time $=30 \mathrm{~s}$ ) using PerkinElmer LS-55 Luminescence spectrometer. Calcein emission was measured at $520 \mathrm{~nm}$ with the excitation wavelength set at $485 \mathrm{~nm}$ using an excitation and emission slit width of $10 \mathrm{~nm}$, each. The percentage of dyeleakage was calculated by normalizing the observed fluorescence intensity at a given time point $\left(\mathrm{F}_{\mathrm{t}}\right)$ with respect to that obtained without addition of ionic liquid $\left(\mathrm{F}_{0, \mathrm{t}}\right)$, and that obtained after addition of $1 \%$ Triton- $\mathrm{X}\left(\mathrm{F}_{\max , \mathrm{t}}\right)$ using following equation

$$
\% \text { Dye leakage }=\frac{F_{t}-F_{0, t}}{F_{\text {max }, t}-F_{t}} \times 100
$$

Separate set of controls $F_{\text {max,t }}$ and $F_{0, t}$ were recorded for each LUV preparation. The normalized data was smoothened by three-point averaging and plotted against time.

Dynamic light scattering (DLS) measurements. Stock solutions of $1 \mathrm{M}\left(\mathrm{C}_{8} \mathrm{MIM}^{+} \mathrm{Br}\right)$, and 100 $\mathrm{mM}\left(\mathrm{C}_{12} \mathrm{MIM}^{+} \mathrm{Br}^{-}\right)$were prepared in $7.7 \mathrm{mM}$ Tris $\mathrm{HCl}$ buffer containing $100 \mathrm{mM}$ of $\mathrm{NaCl}(\mathrm{pH}$ 7.4). Size distribution measurements for POPC and POPG LUVs were performed in the absence and presence of ionic liquids at $25^{\circ} \mathrm{C}$ using Malvern instrument (Zeta sizer, Nano Series, nanoZS, Malvern, U.K.). Samples were thermally equilibrated for $10 \mathrm{~min}$ before each measurement. 
The concentration of lipids in LUVs (POPC or POPG) was fixed at $0.275 \mathrm{mM}$ in each measurement.

Lipid splay measurements using solid-state NMR. Details of sample preparation and ${ }^{1} \mathrm{H}-{ }^{1} \mathrm{H}$ magic-angle-spinning (MAS) solid-state NMR measurements are as described previously. ${ }^{15}$ Briefly, multilamellar vesicles (MLVs) of POPC and POPG containing 0.768 moles of ionic liquid per mole of lipid were packed in $4 \mathrm{~mm}$ high-resolution MAS rotors with spherical Kel-F inserts for NMR measurements. A set of five two dimensional ${ }^{1} \mathrm{H}-{ }^{1} \mathrm{H}$ MAS NOESY spectra ${ }^{25-26}$ were recorded on each sample using mixing times of $0.1,100,200,300$, and 500 ms. NOESY cross-peaks between all lipid segments are observed. As the spin diffusion effect can safely be ruled out for the mixing times used in our study, this is due to the high molecular disorder and mobility of lipid membranes in the liquid crystalline phase. ${ }^{27-28}$ All ${ }^{1} \mathrm{H}$ MAS NMR measurements were carried out on a Bruker Avance III $600 \mathrm{MHz}$ NMR spectrometer using a 4 mm HR-MAS probe at a MAS frequency of $6 \mathrm{kHz}$. The volume of the respective diagonal and cross peaks of $\left(\mathrm{CH}_{2}\right)_{\mathrm{n}}$ - protons in lipid chains and the protons of the lipid head groups were integrated using the Bruker Topspin 3.5 software package. NOE buildup curves were fitted to the spin pair model yielding cross-relaxation rates $(\sigma \mathrm{ij})^{29}$ and used as a measure of lipid splay.

\section{Lipid mixing assay}

Total lipid mixing. Probe dilution assay based on mixing of LUVs containing fluorescence resonance energy transfer (FRET) pairs was used to determine total lipid mixing during membrane fusion. ${ }^{30}$ LUVs containing FRET pair probes NBD-PE (donor) and Rho-PE (acceptor) at a concentration of $1.5 \mathrm{~mol} \%$ each were used. Two sets of LUVs, probe free and probe 
containing, were prepared in $7.7 \mathrm{mM}$ Tris $\mathrm{HCl}$ buffer containing $100 \mathrm{mM}$ of $\mathrm{NaCl}(\mathrm{pH} 7.4)$. Probe containing LUVs were mixed with probe free LUVs at a ratio 1:4 (final lipid concentration was $0.275 \mathrm{mM}$ ) and loaded in a $1 \mathrm{~cm}$ path length quartz cuvette. NBD emission was measured at $530 \mathrm{~nm}$ with the excitation wavelength set at $460 \mathrm{~nm}$ using an excitation and emission slit width of $15 \mathrm{~nm}$, each. A cut off filter at $515 \mathrm{~nm}$ was used between the sample and emission monochromator to avoid scattering interference. Fluorescence dequenching of NBD-PE due to dilution of FRET probes into probe free LUVs was monitored with time as a function of ionic liquid concentration, and used to calculate percentage of total mixing as

$$
\% \text { Total mixing }=\frac{\mathrm{X}_{\mathrm{t}}-\mathrm{X}_{0, \mathrm{t}}}{\mathrm{X}_{\mathrm{max}, \mathrm{t}}-\mathrm{X}_{0, \mathrm{t}}} \times 100
$$

where $X_{t}$ is the fluorescence intensity at time $t, X_{0, t}$ is the fluorescence intensity in the absence of ionic liquid. $\mathrm{X}_{\max , t}$ is the maximum fluorescence intensity (MFI) obtained after addition of $1 \%$ (v/v) Triton X-100. A correction factor of 1.5 was applied to observed fluorescence in the last case as Triton X-100 is known to affect NBD-PE fluorescence. ${ }^{31}$

Lipid mixing in inner (IL) Ionic liquid induced lipid-mixing in the inner and outer monolayers (leaflets) was determined by a modification of the phospholipid-mixing measurement as reported elsewhere. ${ }^{32}$ Next, contribution of IL mixing towards total lipid mixing was determined using selective silencing of OL. Fluorescence intensity of NBD-PE located in the OL of bilayers was completely quenched by treating LUVs containing $1.5 \mathrm{~mol} \%$ of both NBD-PE and Rho-PE with $100 \mathrm{mM}$ sodium dithionite for approximately $1-2 \mathrm{~h}$ on ice in the dark. Sodium dithionite was then removed by the size exclusion chromatography through Sephadex G 50 eluted with a buffer containing $7.7 \mathrm{mM}$ Tris $\mathrm{HCl}$ buffer containing $100 \mathrm{mM}$ of $\mathrm{NaCl}$ (pH 7.4). Percentage IL mixing was calculated as 


$$
\% \mathrm{IL}=\frac{\mathrm{Y}_{\mathrm{t}}-\mathrm{Y}_{0, \mathrm{t}}}{\mathrm{X}_{\mathrm{max}, \mathrm{t}}-\mathrm{Y}_{0, \mathrm{t}}} \times 100
$$

where $Y_{t}$ is the fluorescence intensity at time $t$ in the presence of ionic liquid, $Y_{0, t}$ is the fluorescence intensity in the absence of ionic liquid, and $\mathrm{X}_{\mathrm{max}, \mathrm{t}}$ is MFI as obtained earlier.

Hemifusion \%. Amount of FRET probes in each leaflet is first determined by following the quench kinetics of NBD-PE molecules present in the OL. Fluorescence intensity of LUVs containing $1.5 \mathrm{~mol} \%$ of only NBD-PE was monitored as a function of time after adding $20 \mathrm{mM}$ sodium dithionite (Figure S4). From the fast component of quench kinetics, the percentage $\left(P_{I L}\right)$ of NBD molecules in the IL was found to be $\sim 45 \%$ (in POPC) and $\sim 60 \%$ (in POPG). $\%$ Hemifusion was then calculated by using the difference between the expected IL mixing in the event of full fusion ( $\% \mathrm{IL}_{E X P}=P_{I L} \times \%$ Total mixing) and the observed IL mixing as

$$
\text { Hemifusion } \%=\frac{\% \mathrm{IL}_{E X P}-\% \mathrm{IL}}{\% \mathrm{IL}_{E X P}} \times 100
$$




\section{RESULTS AND DISCUSSIONS}

Imidazolium based ionic liquids are known for their ability to permeabilize the lipid bilayers. ${ }^{15}$, 21, 33 This is mainly studied by observing the fraction of trapped dye leaking out of LUVs as a function of time. These molecules partition themselves into the lipid membrane altering its structural integrity and stability, which results in the leakage of trapped dye. ${ }^{15}$ Figure 1 shows the time dependent leakage of calcein dye trapped inside POPC or POPG LUVs (diameter 120 nm) upon addition of increasing $\left[\mathrm{C}_{12} \mathrm{MIM}\right]^{+} \mathrm{Br}^{-}$concentrations. 


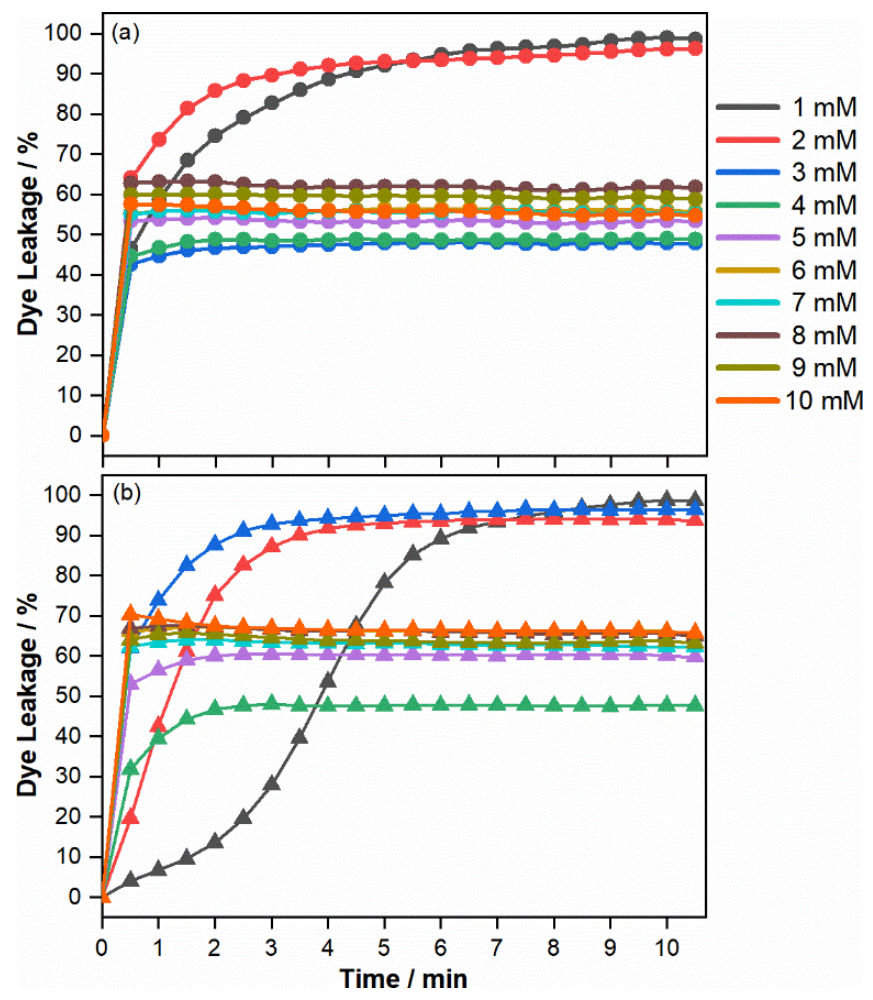

Figure 1. Time course of calcein leakage from (a) POPC, and (b) POPG LUVs upon adding $\left[\mathrm{C}_{12} \mathrm{MIM}\right]^{+} \mathrm{Br}^{-}$in indicated concentrations. Total lipid concentration in LUVs is $0.275 \mathrm{mM}$ in both cases.

An initial increase in $\left[\mathrm{C}_{12} \mathrm{MIM}\right]^{+} \mathrm{Br}^{-}$concentration results in an enhancement of dye leakage kinetics in both cases with maximum dye leakage reaching 100\%. As observed previously, POPC LUVs were found to be more leakage prone as compared to POPG. ${ }^{15,}{ }^{21}$ However, a sudden change in dye leakage profile is observed when $\left[\mathrm{C}_{12} \mathrm{MIM}\right]^{+} \mathrm{Br}^{-}$concentration is increased to $3 \mathrm{mM}$ in POPC, and $4 \mathrm{mM}$ in case of POPG. In both LUVs, maximum dye leakage is reduced to only $48 \%$, accompanied by a sharp enhancement in dye leakage kinetics. These changes could be due to disruption of LUVs as shown by Jing and co-workers where $\left[\mathrm{C}_{12} \mathrm{MIM}\right]^{+} \mathrm{Cl}^{-}$leads to disruption of POPC SUVs when present in concentration exceeding its CMC value. ${ }^{34}$ $\left[\mathrm{C}_{12} \mathrm{MIM}\right]^{+} \mathrm{Br}^{-}$has a $\mathrm{CMC}$ value of $3.3 \mathrm{mM}$ in working buffer (Figure S1), which is fairly close 
to the concentration at which change in leakage profile is observed. Moreover, a complete disruption of LUVs should result in $100 \%$ leakage of dye as observed after the addition of $1 \%$ (v/v) Triton $\mathrm{X}-100$. Even higher $\left[\mathrm{C}_{12} \mathrm{MIM}\right]^{+} \mathrm{Br}^{-}$concentrations yield a similar looking leakage profile with slight enhancement in leakage extent. The fact that dye leakage saturates at value less than $100 \%$ indicates trapping of dye molecules in a stable environment, possibly in a fused vesicle.

Normally, vesicle fusion proceeding through "stalk-hemifusion diaphragm-fusion pore" pathway results in complete mixing of vesicle contents. ${ }^{35}$ However, under some conditions fusion proceeds through a "fusion-through-rupture" pathway which is accompanied by leakage of inner contents. ${ }^{1,35-36}$ This leakage is caused by the formation of fusion-holes close to the stalk where the membrane is relatively thinner. ${ }^{37}$ Leakage rate through these holes depend on the lifetime of holes and diffusion rate of dye molecules, and goes to zero once fusion is complete and the holes are sealed. LUVs studied in this work are composed of cylinder shaped PC and PG lipid molecules which make bilayers with negligible curvature. ${ }^{1}$ Insertion of inverted cone shaped $\left[\mathrm{C}_{12} \mathrm{MIM}\right]^{+}$molecules would disturb the packing of such bilayers. This would enhance the positive curvature in the outer leaflet thereby putting the bilayer under strain. To reduce this additional strain, the bilayer undergoes structural reorganization which results in membrane permeability. ${ }^{15,21}$ However, this mechanism does not seem to be sufficient to rescue the strained bilayers when subjected to high $\left[\mathrm{C}_{12} \mathrm{MIM}\right]^{+}$concentrations. As an additional counter measure, the LUVs could fuse together to form bigger vesicles. These fused vesicles would possess a larger surface area with reduced curvature and hence, lower strain. Dye leakage profiles of POPC and POPG obtained after adding 3 and $4 \mathrm{mM}$ of $\left[\mathrm{C}_{12} \mathrm{MIM}\right]^{+} \mathrm{Br}^{-}$indicate that a similar mechanism underway. In the time it takes for LUVs to fuse, nearly half of the trapped calcein dye (52\% in 
POPC and $52 \%$ in POPG) leaks out through the fusion-holes. As $\left[\mathrm{C}_{12} \mathrm{MIM}\right]^{+}$concentration is increased further, relatively higher amount of dye leaks out in the fusion time period and hence, relatively higher saturation values are observed. These amphiphilic cations have been shown to induce fusion in egg-PC ${ }^{24}$ and DOPC vesicles ${ }^{23}$ previously. However, these studies did not comment on the leaky nature of membrane fusion or formation of fusion-holes.

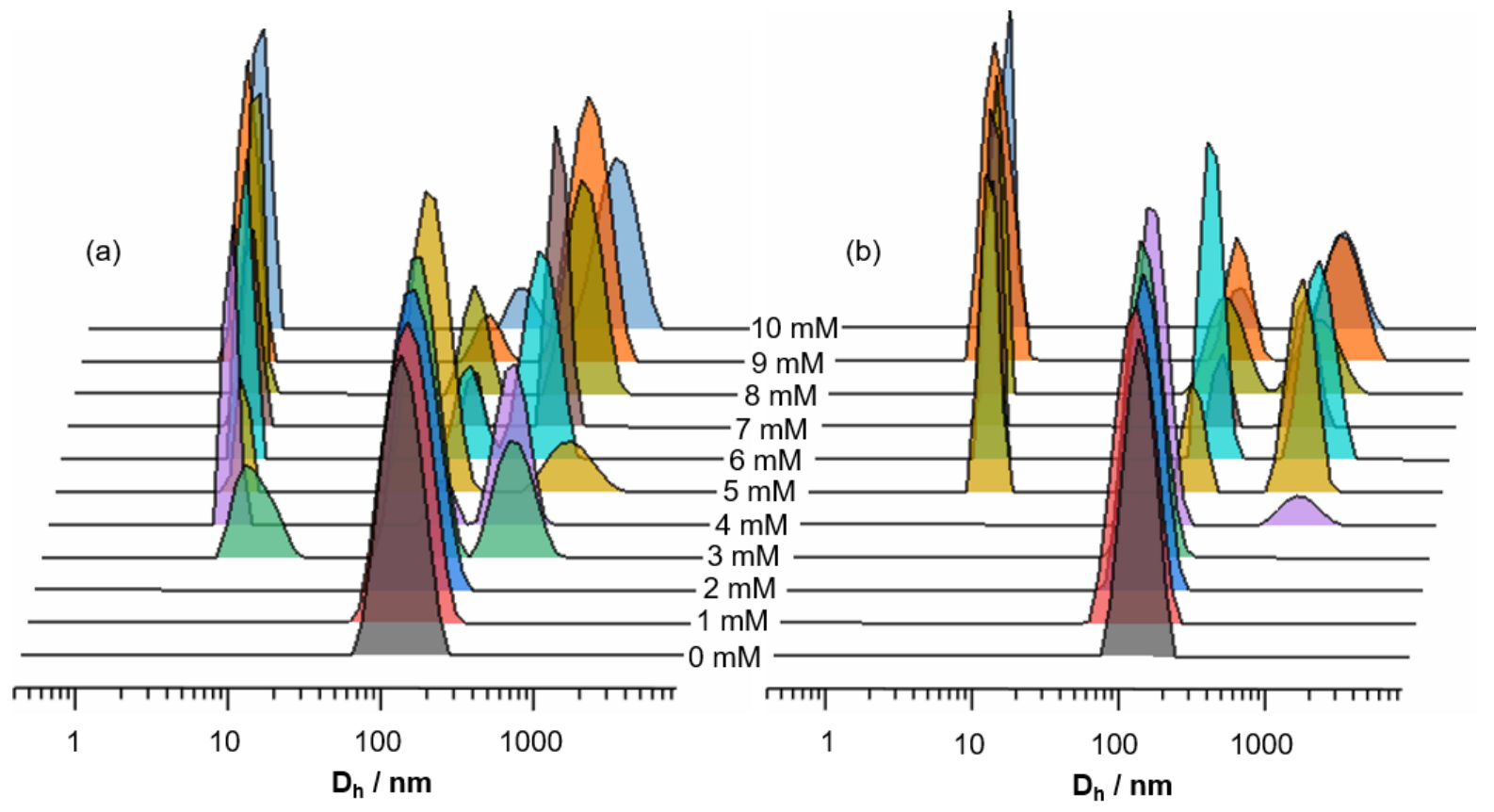

Figure 2. Hydrodynamic radius $\left(\mathrm{D}_{\mathrm{h}}\right)$ of (a) POPC, and (b) POPG LUVs as function of $\left[\mathrm{C}_{12} \mathrm{MIM}\right]^{+} \mathrm{Br}^{-}$concentrations.

To further verify $\left[\mathrm{C}_{12} \mathrm{MIM}\right]^{+} \mathrm{Br}^{-}$induced LUV fusion, we monitored change in size of LUVs using dynamic light scattering measurements (Figure 2). Average diameter of both LUVs is $\sim 120 \mathrm{~nm}$, which remains unchanged in presence of up to 2 and $3 \mathrm{mM}$ of $\left[\mathrm{C}_{12} \mathrm{MIM}\right]^{+} \mathrm{Br}^{-}$in $\mathrm{POPC}$ and POPG LUVs, respectively. As expected, addition of $\left[\mathrm{C}_{12} \mathrm{MIM}\right]^{+} \mathrm{Br}^{-}$in concentrations higher than these results in appearance of a new size distribution peaks centered at $\sim 1110 \mathrm{~nm}$ and $\sim 955$ $\mathrm{nm}$ in POPC and POPG LUVs, respectively. Similar effect was seen in case of mixed DOPC/SM LUVs $(\sim 100 \mathrm{~nm})$ whose size increased up to $1.7 \mu \mathrm{m}$ in presence of 1-ethyl-3-methylimidazolium 
cations due to fusion. ${ }^{23}$ Therefore, peaks centered at $1110 \mathrm{~nm}$ in POPC and $955 \mathrm{~nm}$ in POPG LUVs most likely correspond to large sized fused vesicles. In addition to these peaks, smaller size distributions centered at $\sim 10 \mathrm{~nm}$ were also observed in both DLS profiles. These peaks most likely correspond to mixed $\left[\mathrm{C}_{12} \mathrm{MIM}\right]^{+}$-POPC micelles which are reported to form under these conditions. $^{34}$ These mixed micelles are possibly formed due to interaction of $\left[\mathrm{C}_{12} \mathrm{MIM}\right]^{+}$ micelles with LUVs causing a population exchange between the lipidic and micellar phases. DLS measurements confirm that POPC and POPG LUVs undergo fusion in presence of high $\left[\mathrm{C}_{12} \mathrm{MIM}\right]^{+} \mathrm{Br}^{-}$concentrations. Since changes in DLS and dye leakage profiles take place at same $\left[\mathrm{C}_{12} \mathrm{MIM}\right]^{+} \mathrm{Br}^{-}$concentrations lends further support to the hypothesis that this fusion process is a leaky one due to formation of fusion-holes.
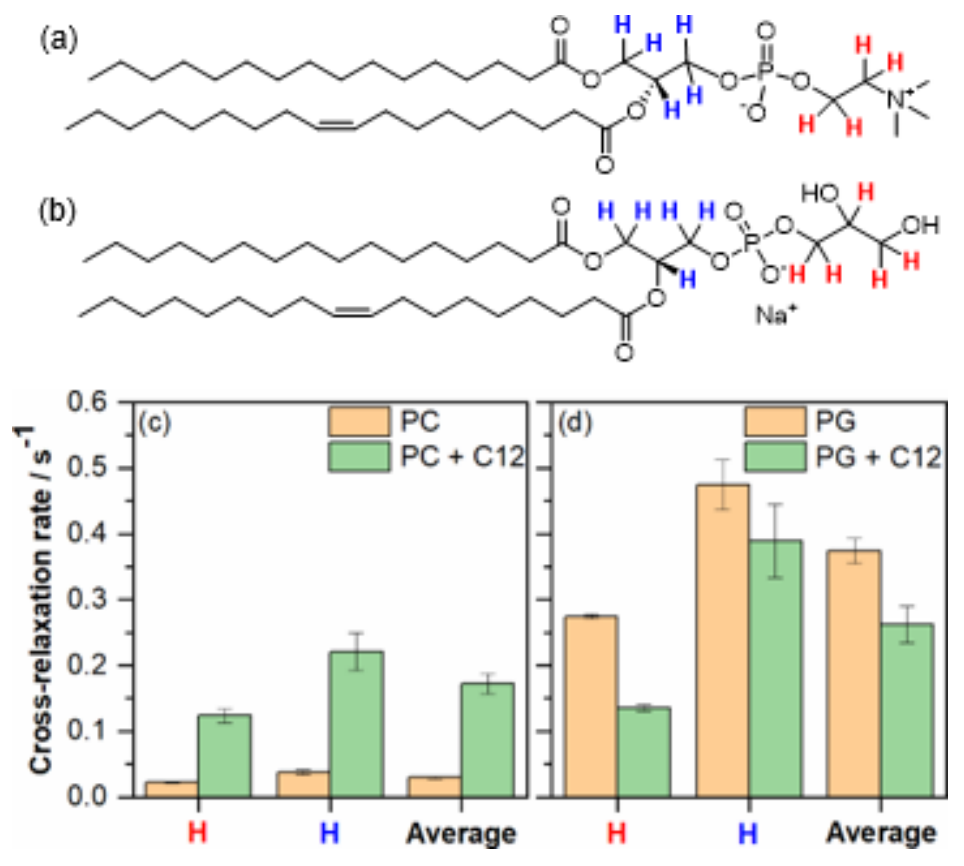

Figure 3. Structures of (a) POPC and (b) POPG molecules with protons in the glycerol and head-group region highlighted in blue and red colors, respectively. ${ }^{1} \mathrm{H}-{ }^{1} \mathrm{H}$ NOESY average cross-relaxation rates between these protons and $-\left(\mathrm{CH}_{2}\right)_{\mathrm{n}^{-}}$protons in lipid chains of (c) POPC 
and (d) POPG observed in the absence and presence of $\left[\mathrm{C}_{12} \mathrm{MIM}\right]^{+} \mathrm{Br}^{-}$. Also shown are the average cross-relaxation rates glycerol and head-group protons taken together.

For two LUVs to fuse, their bilayers must attain a minimal spatial proximity during a collision event by overcoming the electrostatic and hydration barriers between them. ${ }^{38-39}$ A comparison between POPC and POPG tells us that LUVs of former require lower $\left[\mathrm{C}_{12} \mathrm{MIM}\right]^{+} \mathrm{Br}^{-}$ concentrations to fuse. This is counter-intuitive from an electrostatic point of view since $\left[\mathrm{C}_{12} \mathrm{MIM}\right]^{+}$bound POPC LUVs possess a net positive surface charge and hence should be less prone to fusion as compared to near-neutral $\left[\mathrm{C}_{12} \mathrm{MIM}\right]^{+}$bound POPG LUVs (Figure S3). It seems that $\left[\mathrm{C}_{12} \mathrm{MIM}\right]^{+}$induced membrane fusion is initiated by the hydrophobic interaction between the merging bilayers. In absence of $\left[\mathrm{C}_{12} \mathrm{MIM}\right]^{+} \mathrm{Br}^{-}$, POPG bilayers are softer in comparison to POPC due to higher disorder in lipid chains. ${ }^{15}$ Average chain order parameter $(<\mathrm{S}>)$ in pure POPC and POPG membranes is 0.163 and 0.150 , respectively. However, insertion of $\left[\mathrm{C}_{12} \mathrm{MIM}\right]^{+}$molecules renders POPC bilayers more disordered $(<\mathrm{S}>=0.127)$ as compared to POPG $(<\mathrm{S}>=0.154),{ }^{15}$ a factor which could assist membrane fusion through surface exposure of hydrophobic tails thus overcoming the hydration barrier. ${ }^{40}$ Some membrane fusion models have suggested that formation of an initial contact between merging bilayers known as prestalkmembrane. ${ }^{37,41-42}$ Formation of this fusion intermediate is assisted by transient splaying of lipid molecules which exposes the hydrophobic lipid chains to membrane surface. ${ }^{40,43-45}$ Fusogens like membrane-fusogenic soluble peptides ${ }^{46}$ and transmembrane domains ${ }^{47}$ are known to act by promoting the lipid splay phenomenon in bilayers. Keeping this in view, we quantified the impact of $\left[\mathrm{C}_{12} \mathrm{MIM}\right]^{+}$insertion on lipid splay by determining the ${ }^{1} \mathrm{H}-{ }^{1} \mathrm{H}$ NOESY cross-relaxation rates between $-\left(\mathrm{CH}_{2}\right)_{\mathrm{n}^{-}}$protons in the lipid chain and various protons in the glycerol and headgroup region (Figure S5 and S6). As suspected, incorporation of $\left[\mathrm{C}_{12} \mathrm{MIM}\right]^{+}$leads to a very 
significant enhancement in cross-relaxation rates of lipids from $0.0299 \mathrm{~s}^{-1}$ to $0.172 \mathrm{~s}^{-1}$ in POPC bilayers (Figure 3). POPG lipids on the other hand incur a small reduction $(\sim 30 \%)$ upon incorporating $\left[\mathrm{C}_{12} \mathrm{MIM}\right]^{+}$, but yields a value $0.262 \mathrm{~s}^{-1}$ which is still higher than POPC. At the same time, a comparison of average chain order parameter $(<\mathrm{S}>=0.127)$ tells us that $\left[\mathrm{C}_{12} \mathrm{MIM}\right]^{+}$ incorporation makes the POPC lipids chains much more disordered as compared to POPG. This discrepancy could be due to the fact that these distance dependent cross-relaxation rates indicate the probability of contacts between protons and are observed due to conformational disorder of the lipid bilayer which includes lipid splay and lipid fluxes along the bilayer. ${ }^{27,29,48}$ In a nutshell, incorporation of $\left[\mathrm{C}_{12} \mathrm{MIM}\right]^{+}$results in a very large enhancement in lipid splay in POPC which would assist its bilayers in making a hydrophobic contact. When taken together with the fact that $\left[\mathrm{C}_{12} \mathrm{MIM}\right]^{+}$binds more strongly to POPC than POPG, ${ }^{15}$ it explains why membrane fusion occurs at relatively lower $\left[\mathrm{C}_{12} \mathrm{MIM}\right]^{+}$concentrations in case of POPC. 

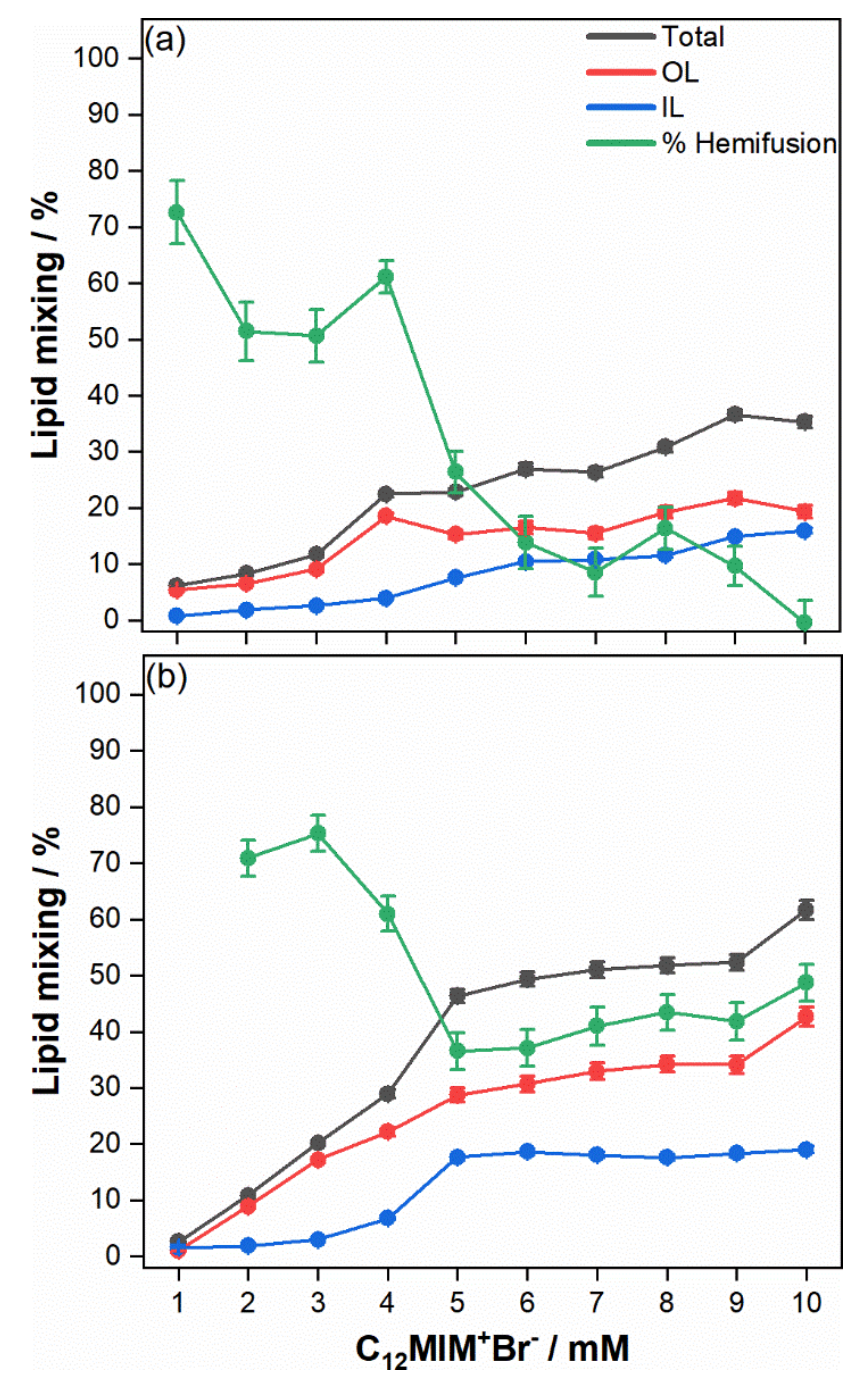

Figure 4. The percentage total lipid mixing (black), OL mixing (red), IL mixing (blue) and hemifused LUVs (green) observed 10 min after addition of $\left[\mathrm{C}_{12} \mathrm{MIM}\right]^{+} \mathrm{Br}^{-}$in (a) $\mathrm{POPC}$, and (b) POPG LUVs.

Membrane fusion involves exchange of lipid populations between the bilayers of merging membranes often termed as lipid mixing. ${ }^{49}$ This property makes it different from simple aggregation of vesicles which also results in large size distributions. Accordingly, we have determined the extent of lipid mixing using the "probe dilution" assay which is particularly insensitive to simple aggregation of LUVs. ${ }^{50}$ In this assay, LUVs containing fluorescently 
labelled lipid probed (NBD-PE and Rho-PE) as FRET pairs were mixed with probe free LUVs and the dilution of fluorescent probes was monitored. The time course of lipid mixing in the POPC or POPG membranes, after the addition of $\left[\mathrm{C}_{12} \mathrm{MIM}\right]^{+} \mathrm{Br}^{-}$ionic liquid, are shown in Figure S7. Figure 4 shows the impact of $\left[\mathrm{C}_{12} \mathrm{MIM}\right]^{+} \mathrm{Br}^{-}$on the extent of lipid mixing 10 minutes after its addition. Even at $1 \mathrm{mM}$, where no $\left[\mathrm{C}_{12} \mathrm{MIM}\right]^{+} \mathrm{Br}^{-}$induced fusion is expected, one observes $\sim 6 \%$ and $\sim 2 \%$ total lipid mixing in POPC and POPG LUVs, respectively. The observed difference is probably due to the fact that more $\left[\mathrm{C}_{12} \mathrm{MIM}\right]^{+}$molecules bind POPC $(\mathrm{K}=590 \pm 60$ $\left.\mathrm{mM}^{-1}\right)$ as compared to POPG $\left(\mathrm{K}=340 \pm 30 \mathrm{mM}^{-1}\right)$ membranes. ${ }^{15}$ In a probe dilution assay, this value will depend on the lateral diffusion of lipids in a bilayer which once again highlights the more dynamic nature of POPC bilayers in presence of $\left[\mathrm{C}_{12} \mathrm{MIM}\right]^{+}$. Subsequent additions of $\left[\mathrm{C}_{12} \mathrm{MIM}\right]^{+} \mathrm{Br}^{-}$results in enhancement of total lipid mixing clearly indicating mixing of bilayers taking place due to LUV fusion. In POPC, total lipid mixing nearly doubles upon increasing $\left[\mathrm{C}_{12} \mathrm{MIM}\right]^{+} \mathrm{Br}^{-}$concentration from 3 to $4 \mathrm{mM}$, followed by an average increase by $\sim 2.5 \%$ up till $10 \mathrm{mM}$. POPG on the other hand shows a much sharper rate of change in total lipid mixing up till $5 \mathrm{mM}\left(\sim 10 \%\right.$ per $\mathrm{mM}$ of $\left.\left[\mathrm{C}_{12} \mathrm{MIM}\right]^{+} \mathrm{Br}\right)$, followed by a slow but gradual increase till $10 \mathrm{mM}$ similar to POPC. As expected, relatively low concentration of $\left[\mathrm{C}_{12} \mathrm{MIM}\right]^{+}$is required to achieve near saturation in lipid mixing of POPC bilayers. In-spite of this, total lipid mixing observed in POPG is nearly two times than in POPC. Since we already know that lipid dynamics in POPC and POPG membranes is not much different after incorporation of $\left[\mathrm{C}_{12} \mathrm{MIM}\right]^{+}$, the possible explanation is that the extent of fusion, i.e. the number of fusing LUVs is higher in case of POPG. Lipid splay determination reveals that $\left[\mathrm{C}_{12} \mathrm{MIM}\right]^{+}$induced membrane fusion of POPC and POPG bilayers is dictated by the hydrophobic interactions. A stronger affinity of $\left[\mathrm{C}_{12} \mathrm{MIM}\right]^{+}$ molecules towards POPC membrane ${ }^{15}$ results in a faster build-up of bilayer strain and hence, an 
early fusion. Though delayed, once the hydrophobic contact is established in POPG, its near neutral LUV carrying a net surface charge of $3.93 \pm 0.49 \mathrm{mV}$ in presence of $4 \mathrm{mM}\left[\mathrm{C}_{12} \mathrm{MIM}\right]^{+}$ undergo rampant fusion (Figure S3). On the other hand, POPC LUVs carrying a net positive surface charge $\left(15.5 \pm 3.3 \mathrm{mV}\right.$ at $\left.3 \mathrm{mM}\left[\mathrm{C}_{12} \mathrm{MIM}\right]^{+}\right)$prevents their close approach. Thus, while hydrophobic factors determine the initiation of $\left[\mathrm{C}_{12} \mathrm{MIM}\right]^{+}$induced fusion, electrostatic factors seem to control its overall extent.

A simple modification of lipid mixing assay allows one to determine the extent of lipid mixing in each individual leaflet, i.e. the outer leaflet (OL) and the inner leaflet (IL) of the bilayer, thus helping us gain further insight in to $\left[\mathrm{C}_{12} \mathrm{MIM}\right]^{+} \mathrm{Br}^{-}$induced fusion pathway. The percentage of IL lipid mixing (in blue, Figure 4) was calculated using selective silencing of the NBD labelled lipids in OL of LUVs in presence of sodium dithionite as explained in material and methods section. The OL lipid mixing (in red, Figure 4) was then calculated as the difference between total lipid and IL mixing. \% IL and \% OL mixing, 10 minutes after addition of $\left[\mathrm{C}_{12} \mathrm{MIM}\right]^{+} \mathrm{Br}^{-}$are shown in Figure 4, and their time dependence are shown in Figure S7. Before the beginning of the fusion, lipid mixing is dominated by OL mixing in both cases. This is probably because insertion of $\left[\mathrm{C}_{12} \mathrm{MIM}\right]^{+}$cations in the OL of POPC and POPG bilayers results in an enhanced lipid dynamics. ${ }^{34}$ After fusion, contribution of IL mixing in POPC shows a steady increase with concentration indicating the increasing dominance of the fully-fused states (\% Hemifusion, Fig. 4). In contrast, OL mixing continues to remain a dominant factor in POPG even at higher concentrations indicating a near-equal contribution of both hemi- and fully-fused states. It is possible that addition of higher $\left[\mathrm{C}_{12} \mathrm{MIM}\right]^{+} \mathrm{Br}^{-}$concentration $(>10 \mathrm{mM})$ in POPG could result in a fully-fused state. A better quantification of hemi-fused vs fully-fused state is provided by a "content mixing assay" which is based on mixing of LUV trapped with $\mathrm{TbCl}_{3}$ (Terbium chloride) 
and DPA (Dipicolinic acid). ${ }^{51}$ However, the leaky nature LUVs due to formation of fusion-holes in the early stage of fusion prevented the application of this assay in our case.

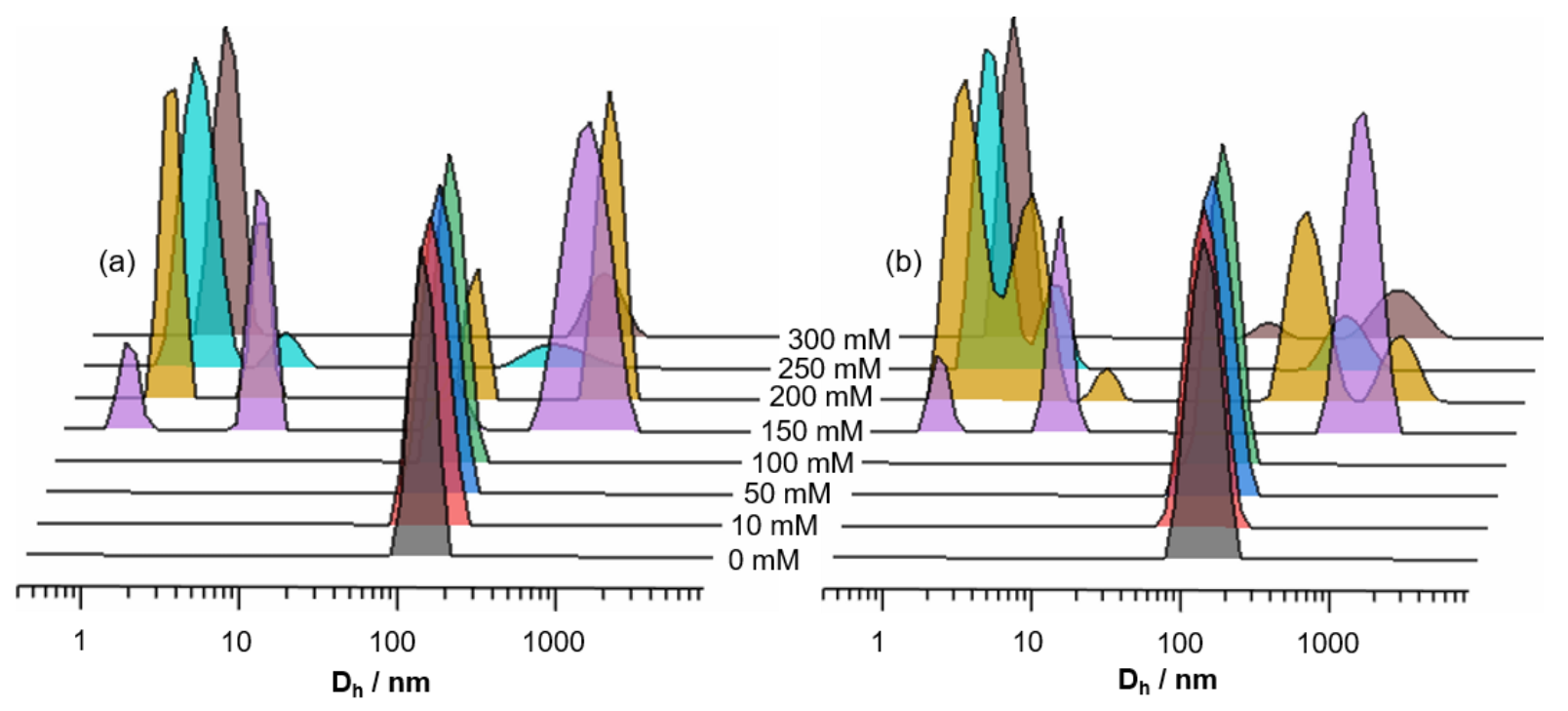

Figure 5. Hydrodynamic radius $\left(D_{h}\right)(a)$ POPC, and (b) POPG LUVs as observed in presence of various $\left[\mathrm{C}_{8} \mathrm{MIM}\right]^{+} \mathrm{Br}^{-}$concentrations.

Our studies have helped us in building a model for membrane fusion induced by ionic liquids containing imidazolium based amphiphilic cations. According to this model, when present in high concentrations (near CMC), $\left[\mathrm{C}_{12} \mathrm{MIM}\right]^{+}$acts as a fusogenic agent for LUVs composed of neutral (POPC; ionic liquid:lipid > 7:1) and negatively charged (POPG; ionic liquid:lipid > 11:1) lipids. While the onset of fusion is critically dependent on $\left[\mathrm{C}_{12} \mathrm{MIM}\right]^{+}$induced lipid splay, its total extent depends on the net surface charge carried by the LUVs. At $\left[\mathrm{C}_{12} \mathrm{MIM}\right]^{+} \mathrm{Br}^{-}$ concentrations studied here, fusion process is dominated by a hemi-fused state and involves formation of leaky fusion-holes in the early stages of fusion. Before concluding our observations, we tested this model using another imidazolium based ionic liquid containing amphiphilic cations with shorter hydrophobic chain $\left(\left[\mathrm{C}_{8} \mathrm{MIM}\right]^{+} \mathrm{Br}^{-}\right)$. In comparison to $\left[\mathrm{C}_{12} \mathrm{MIM}\right]^{+}$, partitioning of $\left[\mathrm{C}_{8} \mathrm{MIM}\right]^{+}$into POPC and POPG bilayers is relatively weak leading to a relatively lower permeabilization at comparable concentrations. ${ }^{15}$ At the same time, insertion of these small 
sized molecules results in large lipid chain disorder and bilayer softness as compared to its long chain counterparts particularly in the POPC membranes. ${ }^{15}$ Figure 5 shows the impact of increasing $\left[\mathrm{C}_{8} \mathrm{MIM}\right]^{+}$concentrations on size of LUVs. As expected, membrane fusion requires much higher $\left[\mathrm{C}_{8} \mathrm{MIM}\right]^{+}$concentrations as compared to $\left[\mathrm{C}_{12} \mathrm{MIM}\right]^{+}$as size distributions corresponding to fused vesicles ( $\sim 825 \mathrm{~nm})$ are observed at $150 \mathrm{mM}$ in both POPC and POPG LUVs. Also observed are the smaller size distributions (in the range of 1-8 $\mathrm{nm}$ ) corresponding to mixed micelles at these concentrations. Lipid mixing assays also confirmed the process of fusion, and like $\left[\mathrm{C}_{12} \mathrm{MIM}\right]^{+}$, extent of fusion was found to be larger in case of POPG (Figure 6a and b). The time course of lipid mixing, OL, and IL mixing in the POPC or POPG membranes, after the addition of $\left[\mathrm{C}_{8} \mathrm{MIM}\right]^{+} \mathrm{Br}^{-}$ionic liquid, are shown in Figure S8. In contrast to $\left[\mathrm{C}_{12} \mathrm{MIM}\right]^{+}, \%$ hemi-fusion is significantly smaller at the onset of fusion. This difference is probably due to a highly disordered nature of POPC $(<\mathrm{S}>=0.116)$ and POPG $(<\mathrm{S}>=0.115)$ lipid chains in presence of $\left[\mathrm{C}_{8} \mathrm{MIM}\right]^{+}$resulting in high membrane fluidity and lipid mixing. ${ }^{15}$ Also, at high IL concentrations (>100 mM) in POPG, IL mixing exceeds OL mixing values resulting in negative $\%$ Hemifusion values. This is possibly due to formation of mixed micelles in presence of abundant $\left[\mathrm{C}_{8} \mathrm{MIM}\right]^{+}$thus providing an additional way to change FRET pair distances apart from the lipid mixing. Figures $\mathbf{6 c}$ and $\mathbf{6 d}$ show the concentration dependent dye leakage profiles of POPC and POPG LUVs, respectively. As expected, a change in leakage kinetics is observed around $\left[\mathrm{C}_{8} \mathrm{MIM}\right]^{+}$concentrations required to induce LUV fusion, confirming its leaky nature due to formation of fusion-holes while the membranes merge together. Lastly, partitioning of $\left[\mathrm{C}_{8} \mathrm{MIM}\right]^{+}$molecules enhanced splaying of POPC lipids by a significant amount ( $0.0299 \mathrm{~s}^{-1}$ to $\left.0.2295 \mathrm{~s}^{-1}\right)$, while increasing it only a little in case of POPG $\left(0.374 \mathrm{~s}^{-1}\right.$ to $\left.0.399 \mathrm{~s}^{-1}\right)$ 
(Figure S9), thus supporting our hypothesis that LUV fusion is driven by hydrophobic interactions.
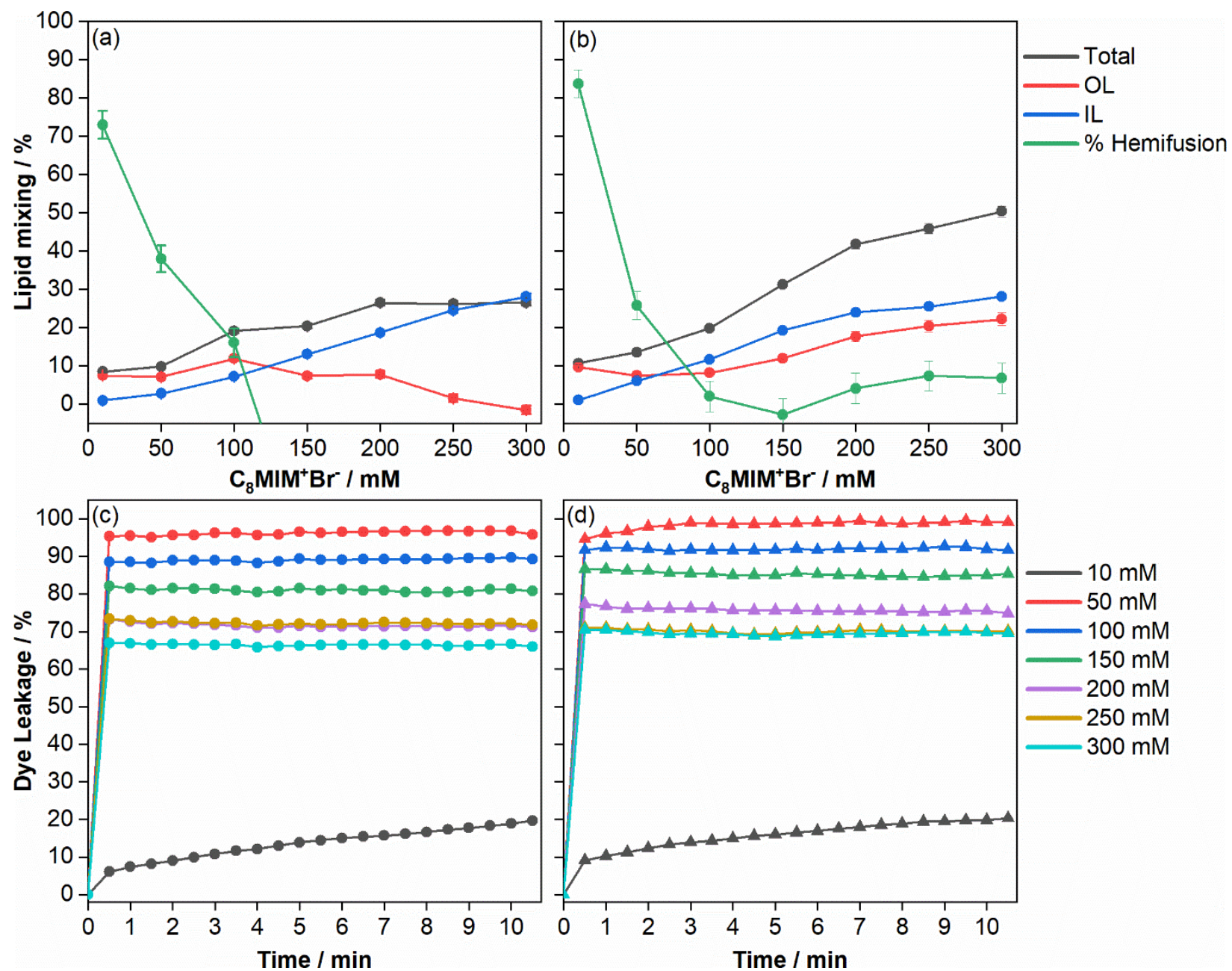

Figure 6. The percentage total lipid mixing (black), OL mixing (red), IL mixing (blue) and hemifused LUVs (green) observed 10 min after addition of $\left[\mathrm{C}_{8} \mathrm{MIM}\right]^{+} \mathrm{Br}^{-}$in (a) POPC, and (b) POPG LUVs. Time course of dye leakage from (c) POPC, and (d) POPG LUVs upon addition of $\left[\mathrm{C}_{8} \mathrm{MIM}\right]^{+} \mathrm{Br}^{-}$in concentration as indicated. 


\section{CONCLUSIONS}

We have conducted a systematic investigation into mechanism of $\left[\mathrm{C}_{\mathrm{n}} \mathrm{MIM}\right]^{+}$induced fusion of POPC and POPG LUVs. The kinetics, extent, and pathway of $\left[\mathrm{C}_{n} \mathrm{MIM}\right]^{+}$induced membrane fusion is critically dependent of lipid dynamics, which is regulated by the interaction of lipid and $\left[\mathrm{C}_{\mathrm{n}} \mathrm{MIM}\right]^{+}$molecules. Insertion of $\left[\mathrm{C}_{\mathrm{n}} \mathrm{MIM}\right]^{+}$molecules alters packing of lipid bilayers, thereby putting them under strain. As a counter measure, lipid chains become more disordered resulting in enhanced exposure of hydrophobic lipid chains to membrane surface through lipid splay. This enables the fusing membrane to overcome the inter-bilayer hydration barrier and make a hydrophobic contact, possibly forming a prestalk-membrane. Accordingly, relatively disordered chains in presence of $\left[\mathrm{C}_{n} \mathrm{MIM}\right]^{+}$cations result in an early onset of fusion in POPC LUVs. Once the hydration barrier is crossed, the extent of fusion is predominantly dictated by electrostatic factors, which favour fusion of near-neutral $\left[\mathrm{C}_{n} \mathrm{MIM}\right]^{+}$inserted POPG LUVs. The fusion process in both membranes seems to proceed through the stalk-hemifusion diaphragm-fusion pore pathway. Early stages of fusion are accompanied by leakage of inner contents from vesicles due to formation of fusion-holes at the stalk intermediate stage. These holes seal off as the membrane evolves into the hemi-fused diaphragm. In a nutshell, our study provides a structure-property model to explain the mechanism of membrane fusion induced by amphiphilic cations in imidazole based ionic-liquids. The outcomes of this model can be used to regulate membrane fusion by varying structural parameters of ionic-liquid components. 


\section{ASSOCIATED CONTENT}

Supporting Information contains $\mathrm{CMC}$ determination of $\mathrm{C}_{12} \mathrm{MIM}^{+} \mathrm{Br}^{-}$and $\mathrm{C}_{8} \mathrm{MIM}^{+} \mathrm{Br}^{-}, \zeta-$ potential measurements, dithionite bleaching, ${ }^{1} \mathrm{H}-{ }^{1} \mathrm{H}$ MAS NOESY spectra, and time dependent lipid mixing profiles of $\mathrm{C}_{12} \mathrm{MIM}^{+} \mathrm{Br}^{-}$and $\mathrm{C}_{8} \mathrm{MIM}^{+} \mathrm{Br}^{-}$, and ${ }^{1} \mathrm{H}-{ }^{1} \mathrm{H}$ MAS NOESY Cross-relaxation rates of POPC and POPG in the presence and absence of $\mathrm{C}_{8} \mathrm{MIM}^{+} \mathrm{Br}^{-}$.

\section{AUTHOR INFORMATION}

Notes

The authors declare no competing financial interests.

\section{Author Contributions}

S.K. synthesized ionic liquids and LUVs, performed dye- leakage and lipid mixing assays, and analyzed ssNMR data. N.K. performed DLS and $\zeta$-potential studies. V.S.M. conceptualized the project, performed ssNMR studies, wrote, reviewed, and edited the manuscript with contributions from all the co-authors.

\section{Orcid Id}

Venus S. Mithu: 0000-0003-2869-9776

Sandeep Kumar: 0000-0002-3867-5586

Navleen Kaur: 0000-0002-4916-5266 


\section{ACKNOWLEDGMENT}

The authors like to acknowledge research funding from Department of Biotechnology, Govt. of India (BT/PR22289/BRB/10/1566/2016). V.S.M. would like to acknowledge German Academic Exchange Service (DAAD) for a research stay grant (ID: 57314018) to perform ssNMR studied in Prof. Daniel Huster's lab at University of Leipzig, Germany. S.K. is thankful

to the Council of Scientific and Industrial Research (CSIR), India, for Senior Research Fellowship (SRF) no. 09/254(0267)/2017- EMR-I. N.K. is thankful to the University Grant Commission (UGC), India, for Senior Research Fellowship (SRF) no. 105743.

\section{REFERENCES}

1. Chernomordik, L. V.; Kozlov, M. M. Mechanics of membrane fusion. Nat. Struct. Mol. Biol. 2008, 15, 675 .

2. Eckert, D. M.; Kim, P. S. Mechanisms of viral membrane fusion and its inhibition. Annu. Rev. Biochem. 2001, 70, 777-810.

3. Duelli, D. and Lazebnik, Y. Cell-to-cell fusion as a link between viruses and cancer. Nat. Rev. Cancer 2007, 7, 968-976.

4. Lentz, B. R.; Malinin, V.; Haque, M. E.; Evans, K. J. Protein machines and lipid assemblies: current views of cell membrane fusion. Curr. Opin. Struct. Biol. 2000, 10, 607-615.

5. Haque, M. E.; Koppaka, V.; Axelsen, P. H.; Lentz, B. R. Properties and structures of the influenza and HIV fusion peptides on lipid membranes: implications for a role in fusion. Biophys. J. 2005, 89, 3183-94. 
6. Kozlov, M. M.; Chernomordik, L. V., A mechanism of protein-mediated fusion: coupling between refolding of the influenza hemagglutinin and lipid rearrangements. Biophys. J. 1998, 75, 1384-96.

7. Mondal Roy, S., Sarkar, M. Membrane fusion induced by small molecules and ions. J. Lipids 2011, 2011.

8. Goodall, A. H.; Galloway, M. J.; Fisher, D.; Lucy, J. A. The interactions of dispersions of lipid-soluble fusogens with hen erythrocytes. Portland Press Limited 1979, 937-939.

9. Mason, W.; Miller, N. G. A. Fusion of charged and uncharged liposomes by n-alkyl bromides. Biochem. Biophys. Res. Commun. 1979, 91, 878-885.

10. Maggio, B.; Lucy, J. A. Interactions of water- soluble fusogens with phospholipids in monolayers. FEBS Lett. 1978, 94, 301-304.

11. Cevc, G. Membrane electrostatics. Biochim. Biophys. Acta, Biomembr. 1990, 1031, 311-382.

12. Welton, T. Room-temperature ionic liquids. Solvents for synthesis and catalysis. Chem. Rev. 1999, 99, 2071-2084.

13. Egorova, K. S.; Gordeev, E. G.; Ananikov, V. P. Biological activity of ionic liquids and their application in pharmaceutics and medicine. Chem. Rev. 2017, 117, 7132-7189.

14. Pham, T. P. T.; Cho, C. W.; Yun, Y. S. Environmental fate and toxicity of ionic liquids: a review. Water Res. 2010, 44, 352-372. 
15. Kumar, S.; Scheidt, H. A.; Kaur, N.; Kang, T. S.; Gahlay, G. K.; Huster, D.; Mithu, V. S. Effect of the Alkyl Chain Length of Amphiphilic Ionic Liquids on the Structure and Dynamics of Model Lipid Membranes. Langmuir 2019, 35, 12215-12223.

16. Bingham, R. J.; Ballone, P. Computational study of room-temperature ionic liquids interacting with a POPC phospholipid bilayer. J. Phys. Chem. B 2012, 116, 11205-11216.

17. Yoo, B.; Shah, J. K.; Zhu, Y.; Maginn, E. J. Amphiphilic interactions of ionic liquids with lipid biomembranes: a molecular simulation study. Soft Matter 2014, 10, 8641-8651.

18. Benedetto, A.; Ballone, P. Engineering, Room temperature ionic liquids meet biomolecules: a microscopic view of structure and dynamics. ACS Sustainable Chem. Eng. 2015, 4, 392412.

19. Benedetto, A.; Heinrich, F.; Gonzalez, M. A.; Fragneto, G.; Watkins, E.; Ballone, P. Structure and stability of phospholipid bilayers hydrated by a room-temperature ionic liquid/water solution: a neutron reflectometry study. J. Phys. Chem. B 2014, 118, $12192-$ 12206.

20. Sharma, V.K.; Mukhopadhyay, R. Deciphering interactions of ionic liquids with biomembrane. Biophys. Rev. 2018, 10, 721-734.

21. Kumar, S.; Scheidt, H. A.; Kaur, N.; Kaur, A.; Kang, T. S.; Huster, D.; Mithu, V. S. Amphiphilic Ionic Liquid-Induced Membrane Permeabilization: Binding Is Not Enough. $J$. Phys. Chem. B 2018, 122, 6763-6770. 
22. Yoo, B.; Zhu, Y.; Maginn, E. J. Molecular mechanism of ionic-liquid-induced membrane disruption: morphological changes to bilayers, multilayers, and vesicles. Langmuir 2016, 32, $5403-5411$.

23. Hayakawa, E. H.; Mochizuki, E.; Tsuda, T.; Akiyoshi, K.; Matsuoka, H.; Kuwabata, S. The effect of hydrophilic ionic liquids 1-ethyl-3-methylimidazolium lactate and choline lactate on lipid vesicle fusion. Plos one 2013, 8, e85467.

24. Galletti, P.; Malferrari, D.; Samorì, C.; Sartor, G.; Tagliavini, E. Effects of ionic liquids on membrane fusion and lipid aggregation of egg-PC liposomes. Colloids Surf., B 2015, 125, $142-150$.

25. Jeener, J.; Meier, B.; Bachmann, P.; Ernst, R. R., Investigation of exchange processes by two- dimensional NMR spectroscopy. J. Chem. Phys. 1979, 71, 4546-4553.

26. Kumar, A.; Wagner, G.; Ernst, R.R.; Wuethrich, K. Buildup rates of the nuclear Overhauser effect measured by two-dimensional proton magnetic resonance spectroscopy: implications for studies of protein conformation. J. Am. Chem. Soc., 1981, 103, 3654-3658.25.

27. Huster, D.; Arnold, K.; Gawrisch, K. Investigation of lipid organization in biological membranes by two-dimensional nuclear Overhauser enhancement spectroscopy. J. Phys. chem. B 1999, 103, 243-251.

28. Huster, D.; and Gawrisch, K. NOESY NMR crosspeaks between lipid headgroups and hydrocarbon chains: spin diffusion or molecular disorder? J. Am. Chem. Soc. 1999, 121, 1992-1993. 
29. Scheidt, H. A.; Huster, D. The interaction of small molecules with phospholipid membranes studied by ${ }^{1}$ H NOESY NMR under magic-angle spinning. Acta Pharmacol. Sin. 2008, 29, 35.

30. Struck, D. K.; Hoekstra, D.; Pagano, R. E. Use of resonance energy transfer to monitor membrane fusion. Biochemistry 1981, 20, 4093-4099.

31. Torchilin, V.; Weissig, V. Liposomes: a practical approach. Oxford University Press. 2003, 264.

32. Meers, P.; Ali, S.; Erukulla, R.; Janoff, A. S. Novel inner monolayer fusion assays reveal differential monolayer mixing associated with cation-dependent membrane fusion. Biochim. Biophys. Acta, Biomembr. 2000, 1467, 227-243.

33. Evans, K. O. Physicochemical, S. A.; Aspects, E., Room-temperature ionic liquid cations act as short-chain surfactants and disintegrate a phospholipid bilayer. Colloids Surf., A 2006, 274, 11-17.

34. Jing, B.; Lan, N.; Qiu, J.; Zhu, Y. Interaction of ionic liquids with a lipid bilayer: a biophysical study of ionic liquid cytotoxicity. J. Phys. Chem. B 2016, 120, 2781-2789.

35. Frolov, V.; Dunina-Barkovskaya, A. Y.; Samsonov, A.; Zimmerberg, J. Membrane permeability changes at early stages of influenza hemagglutinin-mediated fusion. Biophys. $J$. 2003, $85,1725-1733$.

36. Bu, B.; Crowe, M.; Diao, J.; Ji, B.; Li, D. Cholesterol suppresses membrane leakage by decreasing water penetrability. Soft matter 2018, 14, 5277-5282. 
37. Kozlovsky, Y.; Chernomordik, L. V.; Kozlov, M. M. Lipid intermediates in membrane fusion: formation, structure, and decay of hemifusion diaphragm. Biophys. J. 2002, 83, 2634-2651.

38. Burgess, S. W.; McIntosh, T. J.; Lentz, B. R. Modulation of poly (ethylene glycol)-induced fusion by membrane hydration: importance of inter bilayer separation. Biochemistry 1992, $31,2653-2661$.

39. Ohki, S. A mechanism of divalent ion-induced phosphatidylserine membrane fusion. Biochim. Biophys. Acta, Biomembr. 1982, 689, 1-11.

40. Smirnova, Y. G.; Marrink, S. J.; Lipowsky, R.; Knecht, V. Solvent-exposed tails as prestalk transition states for membrane fusion at low hydration. J. Am. Chem. Soc. 2010, 132, 67106718.

41. Kozlovsky, Y.; Efrat, A.; Siegel, D. A.; Kozlov, M. M. Stalk phase formation: effects of dehydration and saddle splay modulus. Biophys. J. 2004, 87, 2508-2521.

42. Kozlovsky, Y.; Kozlov, M. M. Stalk model of membrane fusion: solution of energy crisis. Biophys. J. 2002, 82, 882-895.

43. Kinnunen, P. K.; Holopainen, J. M. Mechanisms of initiation of membrane fusion: role of lipids. Biosci. Rep. 2000, 20, 465-482.

44. Mirjanian, D.; Dickey, A. N.; Hoh, J. H.; Woolf, T. B.; Stevens, M. J. Splaying of aliphatic tails plays a central role in barrier crossing during liposome fusion. J. Phy. Chem. B 2010, 114, 11061-11068. 
45. Kawamoto, S.; Shinoda, W. Free energy analysis along the stalk mechanism of membrane fusion. Soft matter 2014, 10, 3048-3054.

46. Larsson, P.; Kasson, P. M. Lipid tail protrusion in simulations predicts fusogenic activity of influenza fusion peptide mutants and conformational models. Plos one Comput. Biol. 2013, 9.

47. Scheidt, H. A.; Kolocaj, K.; Veje Kristensen, J.; Huster, D.; Langosch, D. Transmembrane helix induces membrane fusion through lipid binding and splay. J. Phys. Chem. Lett. 2018, 9, 3181-3186.

48. Mihailescu, M.; Vaswani, R. G.; Jardón-Valadez, E.; Castro-Román, F.; Freites, J. A.; Worcester, D. L.; Chamberlin, A. R.; Tobias, D. J.; White, S. H. Acyl-chain methyl distributions of liquid-ordered and-disordered membranes. Biophys. J. 2011, 100, 1455-1462

49. Cevc, G.; Richardsen, H. Lipid vesicles and membrane fusion. Adv. Drug Deliv. Rev 1999, $38,207-232$.

50. Duzgunes, N.; Allen, T. M.; Fedor, J.; Papahadjopoulos, D. Lipid mixing during membrane aggregation and fusion: why fusion assays disagree. Biochemistry 1987, 26, 8435-8442.

51. Wilschut, J.; Duzgunes, N.; Fraley, R.; Papahadjopoulos, D. Studies on the mechanism of membrane fusion: kinetics of calcium ion induced fusion of phosphatidylserine vesicles followed by a new assay for mixing of aqueous vesicle contents. Biochemistry 1980, 19, 6011-6021. 